\title{
Quantum disorder in the two-dimensional pyrochlore Heisenberg antiferromagnet
}

\author{
S. E. Palmer ${ }^{1,2}$ and J. T. Chalker ${ }^{1}$ \\ ${ }^{1}$ Theoretical Physics, University of Oxford, 1 Keble Road, Oxford OX1 3NP, UK \\ ${ }^{2}$ Current address: Sloan Center for Theoretical Neuroscience, University of California at San Francisco, Box 0444 , \\ 513 Parnassus Avenue, San Francisco, CA 94143, USA
}

\begin{abstract}
We present the results of an exact diagonalization study of the spin-1/2 Heisenberg antiferromagnet on a two-dimensional version of the pyrochlore lattice, also known as the square lattice with crossings or the checkerboard lattice. Examining the low energy spectra for systems of up to 24 spins, we find that all clusters studied have non-degenerate ground states with total spin zero, and big energy gaps to states with higher total spin. We also find a large number of non-magnetic excitations at energies within this spin gap. Spin-spin and spin-Peierls correlation functions appear to be short-ranged, and we suggest that the ground state is magnetically disordered.
\end{abstract}

\section{INTRODUCTION}

Geometrically frustrated magnets have attracted the interest of both experimentalists and theorists in recent years [1], particularly because of their unusual lowtemperature properties. Fluctuations enhanced by frustration can destroy long range order, giving rise to disordered ground states even in systems with two or three space dimensions. Present understanding of such states remains incomplete. In this context it is useful to study a variety of models, so as to establish the types of behavior that are possible and to isolate good instances of each type.

Examples of geometrically frustrated lattices include the triangular and kagomé lattices in two dimensions, and the pyrochlore lattice in three dimensions. All these are based on frustrated units - triangles in two dimensions and tetrahedra in three dimensions - and in the last two examples these units are combined in a sitesharing arrangement. Properties of the spin- $1 / 2$ Heisenberg antiferromagnet with nearest-neighbor interactions have been studied for each of these lattices. In the case of the triangular lattice, the three sublattice Néel order of the classical ground state is believed to be stable against quantum fluctuations [2.3]. By contrast, in the case of the spin-1/2 kagomé Heisenberg antiferromagnet (KHAF), analysis of the low-energy spectra for finite clusters in-

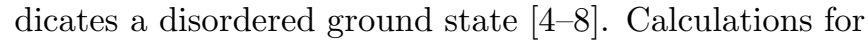
the three-dimensional spin-1/2 pyrochlore Heisenberg antiferromagnet present a great challenge; an approximate approach [9] yields a disordered ground state in that case, too.

In this paper, we describe properties of the spin- $1 / 2$ Heisenberg antiferromagnet on a two-dimensional version of the pyrochlore lattice, also known as the square lattice with crossings or the checkerboard lattice. We denote this model by SLWC. The lattice is illustrated in Fig. 1. The frustrated plaquettes (those with bondcrossings) have the connectivity of tetrahedra, and are corner-sharing, as in the three-dimensional pyrochlore lattice. The classical Heisenberg model on this two-

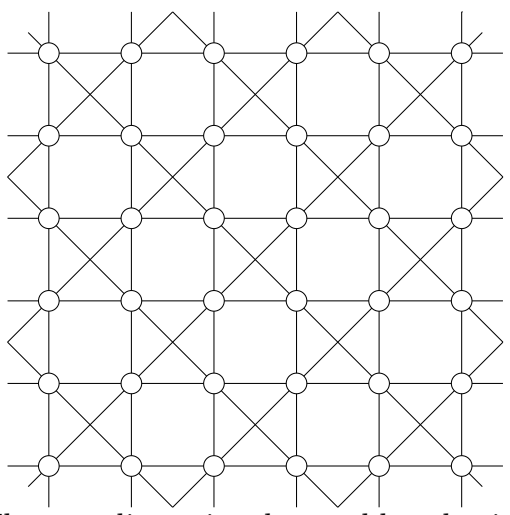

FIG. 1. The two-dimensional pyrochlore lattice, or square lattice with crossings. Circles indicate spins of the Heisenberg model, and lines denote exchange interactions, all of equal strength.

dimensional lattice is known to have disordered ground states [10], while for the quantum Heisenberg model with general spin $S$ it has been proved [11] that all ground states have total spin zero. From calculations presented below of the low-energy spectra for clusters of $N$ sites, with $N$ taking even values from $N=10$ to $N=24$, we conclude that the ground state is disordered at $S=1 / 2$.

The SLWC joins a rather short list of two-dimensional Heisenberg spin systems known to have disordered quantum ground states. Other examples, apart from the KHAF, involve frustration which is not geometric but arises either from further neighbor interactions or from multiple spin exchange.

The effects of further-neighbor interactions have been studied extensively for the Heisenberg model on the square lattice. With nearest-neighbor exchange $J_{1}$ and second neighbor exchange $J_{2}$, Néel order is destabilized by large ground state degeneracy in the classical model at an exchange ratio of $J_{1} / J_{2}=2$ [12]. With spin- $1 / 2$, it is likely that the ground state is magnetically disordered 
and has a gap for all excitations [13 22]. The SLWC and the $J_{1}-J_{2}$ model at $J_{1} / J_{2}=2$ are quite closely related, in the sense that the $J_{1}-J_{2}$ model has second-neighbor exchange interactions which, compared to those in the SLWC, are twice as numerous but of half the strength. It has been noted very recently [23] that a linear spinwave analysis suggests a disordered ground state for the SLWC, as in the $J_{1}-J_{2}$ model.

The consequences of frustration by multiple spin exchange have been examined for the spin-1/2 Heisenberg antiferromagnet on the triangular lattice 24 26]. Competition between two-spin and four-spin exchange leads to two disordered spin states: one has an energy gap for excitations, while the other is similar to the KHAF in having many low-energy excitations.

\section{MODEL AND METHODS}

The Hamiltonian we study is

$$
\mathcal{H}=2 \sum_{\langle i, j\rangle} \mathbf{S}_{i} \cdot \mathbf{S}_{j},
$$

where the sum is taken over pairs of spins $i$ and $j$ joined by bonds on the lattice of Fig. 1 1 and the $\mathbf{S}_{i}$ are spin- $1 / 2$ operators. The magnitude of the exchange interaction in this Hamiltonian is the same as that used for the KHAF in Ref. [7], and is a factor of two larger than that used in References [4] and [6].

We may also express the Hamiltonian as a sum over tetrahedra, equivalent to square plaquettes with crossings, by writing

$$
\mathcal{H}=\sum_{\alpha}\left[\left(\mathbf{J}_{\alpha}\right)^{2}-3\right]
$$

where $\alpha$ labels the frustrated plaquettes and $\mathbf{J}_{\alpha}=\mathbf{S}_{a}+$ $\mathbf{S}_{b}+\mathbf{S}_{c}+\mathbf{S}_{d}$ is the total spin of plaquette $\alpha$, to which the sites $a, b, c$ and $d$ belong. Expressing the eigenvalues of $\left|\mathbf{J}_{\alpha}\right|^{2}$ as $J(J+1)$, the quantum number $J$ takes the values 0,1 or 2 . Since each spin is a member of two plaquettes with crossings, the operators $\mathbf{J}_{\alpha}$ are not all independent. In consequence, it is not possible to construct a state in which $J=0$ for every $\alpha$. Nevertheless, we show below that $J=0$ dominates and that $J=2$ has very small weight.

We use symmetry under spin rotations to blockdiagonalize the Hamiltonian, and employ the Lanczos algorithm to calculate the low-lying eigenvalues for clusters of up to 24 spins. We find eigenvectors by inverse iteration. We test the accuracy of an eigenvalue obtained after $j$ steps of the Lanczos algorithm by performing an additional $j / 10$ steps and then recalculating the eigenvalue: we report only results for which the eigenvalue estimate changes by less than $10^{-6}$ under this test.

As a check of the code, we study 12 and 21 site clusters for the spin- $1 / 2$ triangular lattice Heisenberg antiferromagnet and the KHAF, examined previously in References [27] and [4. We reproduce the energy levels and

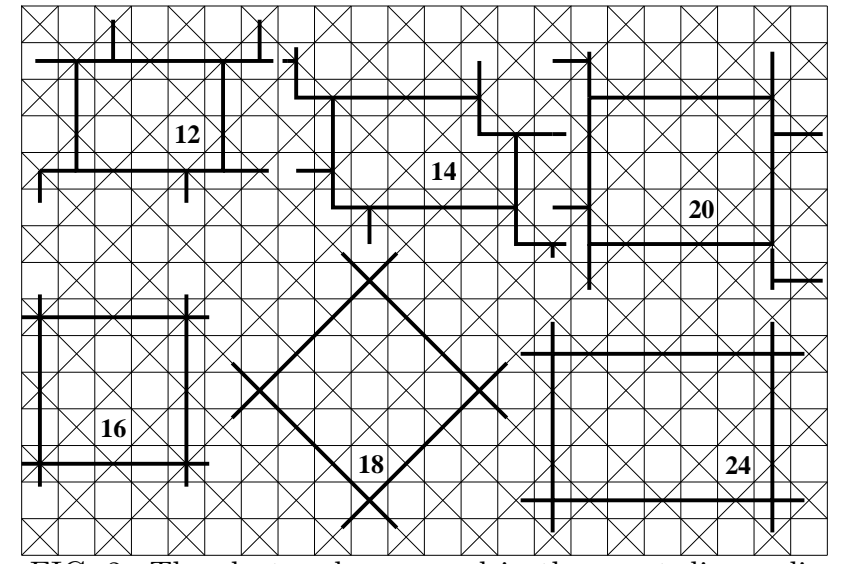

FIG. 2. The cluster shapes used in the exact diagonalization calculations.

degeneracies to the accuracy reported in each of those papers.

The clusters we study for the SLWC are shown in Fig. 2. For some cluster sizes, several inequivalent shapes are possible. In these cases we choose the shape for which the ground state energy is lowest, on the basis that these shapes presumably have the least frustrating boundary conditions. In particular (with the exception of the 14site cluster) the clusters of Fig. 2 leave unfrustrated the two antiferromagnetically ordered states found in the $J_{1}$ $J_{2}$ model, at small and large $J_{2} / J_{1}$ respectively [12].

\section{RESULTS}

\section{A. Energy level spectra}

The low-energy spectra for the $16,18,20$, and 24 site clusters are displayed in Figures 3 and 1 , labeled by the total spin quantum number, $S$. They are also listed in Table If. We notice several distinctive features of these results. First, the ground state for each cluster is nondegenerate, and has total spin zero as expected from the rigorous results of Ref. [11]. Second, there is no evidence in the spectra of the structure which one would expect if the system had Néel order in the thermodynamic limit. More specifically, for a Néel-ordered system the quantum mechanics of the order parameter gives rise to a set of low-lying energy levels [28 33], termed quasi-degenerate joint states in recent studies [2, 7]. These are separated in energy from other states and have an excitation energy that varies with the total spin quantum number $S$ and the cluster size $N$ as $S(S+1) / N$. States of this kind are clearly identifiable in the spectra of triangular lattice Heisenberg antiferromagnet clusters from the same size range [2]. Their absence in the SLWC suggests that the ground state of the SLWC does not possess Néel order. Third, as we discuss below in more detail, the number of 


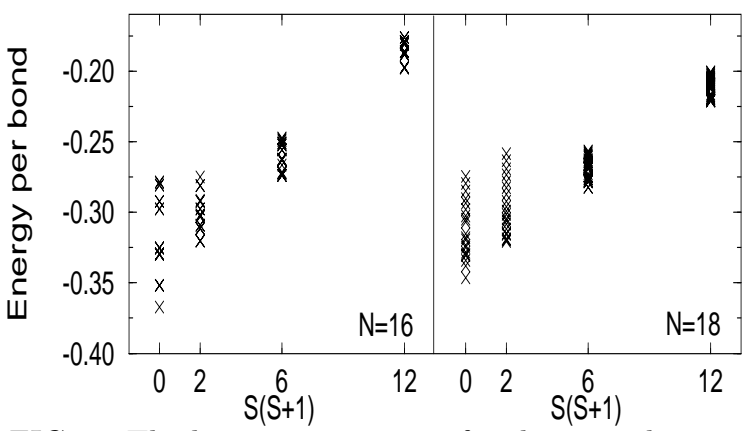

FIG. 3. The low energy spectra for the 16 and 18 site clusters for the SLWC.

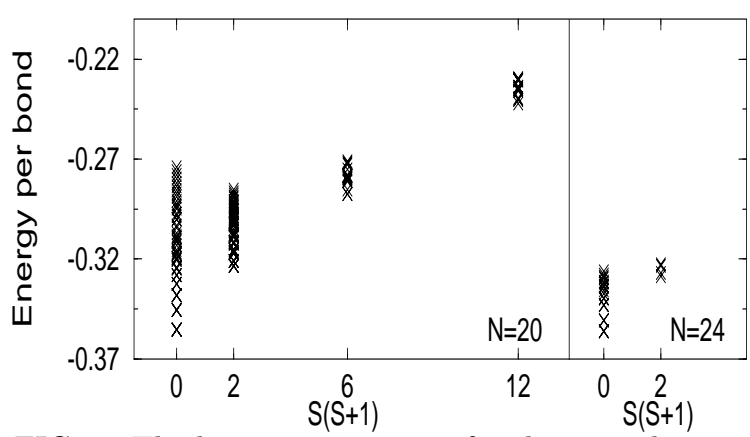

FIG. 4. The low energy spectra for the 20 and 24 site clusters for the SLWC.

states lying within the triplet spin gap is large and increases with cluster size. Interestingly, both the absence of quasi-degenerate states and the presence of low-lying singlet excitations are also features of the KHAF [7],8].

We comment in passing that the data of Table Iinclude some initially unexpected degeneracies for excited states, involving factors of 3 and 5. A 3-fold degeneracy on the 16 -site cluster can be understood by recalling that each crossed square has the same connectivity as a tetrahedron, which has four axes of 3-fold rotational symmetry. Analogously, cycles of length 3 arise from permutations of the tetrahedra in the 16 site cluster. We believe that other 3 -fold degeneracies appear in a similar way, and that 5 -fold degeneracies on the 20 -site cluster are associated with translations.

\section{B. Dependence on cluster size}

The variation with cluster size of the ground state energy per spin is illustrated in Fig. .5. The unit cell of the lattice contains two sites, and so it is natural to group clusters with even and odd numbers of unit cells separately. Doing this, a common extrapolation to the infinite system can be found, as shown. The ground state energy as well as the triplet and singlet gaps are plotted versus $1 / N$. (This choice is made simply as a convenient
TABLE I. Lowest eigenvalues given as an average energy per bond, total spin, $S$, and degeneracies for various sample sizes on the square lattice with crossings. A '-' indicates that the degeneracies of these levels have not been calculated. Degeneracies do not include the $2 S+1 m_{S}$ degeneracy.

\begin{tabular}{|c|c|c|c|}
\hline$N$ & $\overline{2\left\langle\mathbf{S}_{i} \cdot \mathbf{S}_{j}\right\rangle}$ & $S$ & deg. \\
\hline \multirow[t]{6}{*}{16} & -0.367045 & 0 & 1 \\
\hline & -0.351695 & 0 & 4 \\
\hline & -0.329769 & 0 & 4 \\
\hline & -0.329403 & 0 & 12 \\
\hline & -0.325200 & 0 & 6 \\
\hline & -0.320232 & 1 & 9 \\
\hline \multirow[t]{9}{*}{$\overline{18}$} & -0.346423 & 0 & 1 \\
\hline & -0.337917 & 0 & 4 \\
\hline & -0.334194 & 0 & 1 \\
\hline & -0.330624 & 0 & 4 \\
\hline & -0.329751 & 0 & 1 \\
\hline & -0.328540 & 0 & 1 \\
\hline & -0.324801 & 0 & 8 \\
\hline & -0.323410 & 0 & 4 \\
\hline & -0.320797 & 1 & - \\
\hline \multirow[t]{8}{*}{$\overline{20}$} & -0.359897 & 0 & 1 \\
\hline & -0.350021 & 0 & 2 \\
\hline & -0.342692 & 0 & 2 \\
\hline & -0.336797 & 0 & 5 \\
\hline & -0.332620 & 0 & 5 \\
\hline & -0.329264 & 0 & 5 \\
\hline & -0.328645 & 0 & 5 \\
\hline & -0.327938 & 1 & - \\
\hline \multirow[t]{15}{*}{$\overline{24}$} & -0.360776 & 0 & 1 \\
\hline & -0.355258 & 0 & 1 \\
\hline & -0.347472 & 0 & 2 \\
\hline & -0.344195 & 0 & 1 \\
\hline & -0.344142 & 0 & 1 \\
\hline & -0.341941 & 0 & 6 \\
\hline & -0.340329 & 0 & 1 \\
\hline & -0.338475 & 0 & 2 \\
\hline & -0.337994 & 0 & 6 \\
\hline & -0.337811 & 0 & 6 \\
\hline & -0.336385 & 0 & 1 \\
\hline & -0.335848 & 0 & 2 \\
\hline & -0.334265 & 0 & 6 \\
\hline & -0.333261 & 0 & 3 \\
\hline & -0.332998 & 1 & - \\
\hline
\end{tabular}




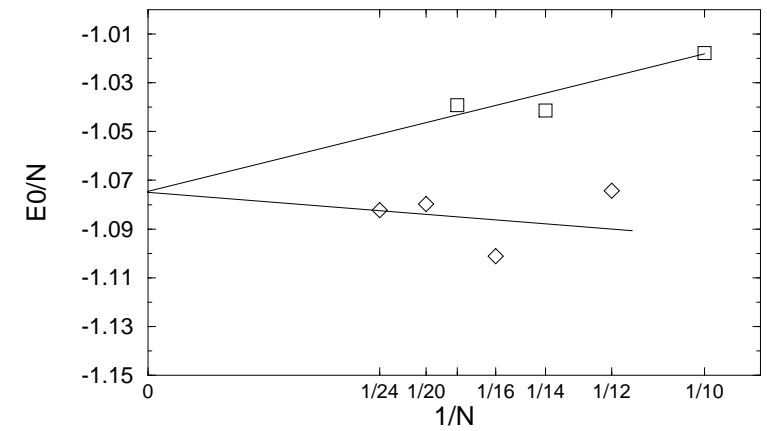

FIG. 5. The ground state energy per spin plotted versus $1 / N$, where $N$ is the number of spins the system. The two lines shown are guides to the eye and are drawn through the data for the larger cluster sizes, with an even (diamonds) and odd (squares) number of unit cells respectively.

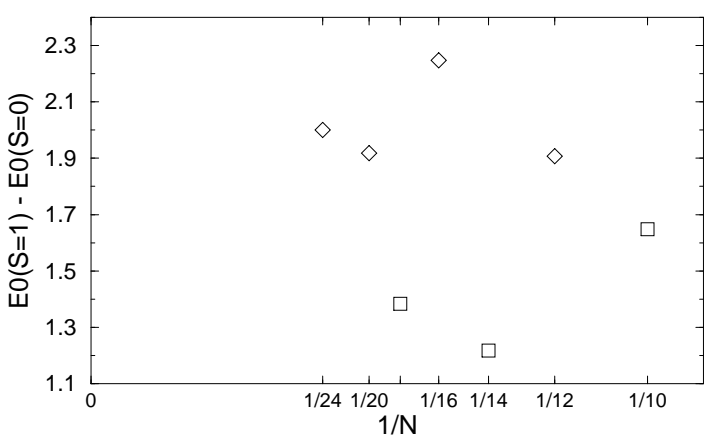

FIG. 6. The gap to the first excited state with higher total spin plotted versus inverse system size.

way of separating points along the horizontal axis.) The cluster sizes studied here are small, and there are clear difficulties in drawing quantitative conclusions from the data. Nevertheless, a definite indication emerges that the ground state is magnetically disordered.

The dependence of the spin gap on cluster size is presented in Fig. 6 . The fact that the spin gap appears to remain non-zero in the thermodynamic limit is, of course, in contrast to the size-dependence of the longest wavelength magnon energy in a system with Néel order. It suggests again that the SLWC does not have Néel order. A non-zero spin gap is also believed to be a feature of the $\mathrm{KHAF}$, but the spin gap for the KHAF is substantially smaller than that measured for the SLWC. For example, for the 21 site cluster on the kagomé lattice, Leung and Elser report a spin gap of size 0.5573 [6] (scaled to accommodate our factor of 2 in the Hamiltonian), compared to a spin gap of size 2.00 for our 24 site cluster. Similarly, the $J_{1}-J_{2}$ model at $J_{1} / J_{2}=2$ has a non-zero spin gap, but one that is smaller by a factor of about 2 at $N=24$ than for the SLWC [19].

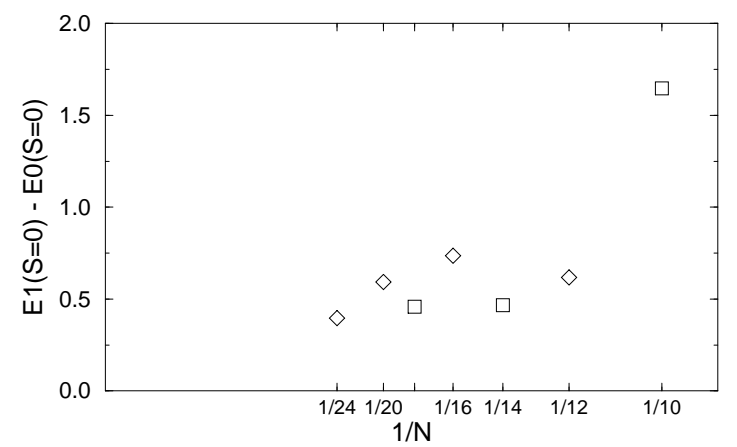

FIG. 7. The gap to the first excited state above the ground state is plotted versus inverse system size. For all clusters, the first excited state is also a singlet state.

\section{Moments of $\mathbf{J}_{\alpha}^{2}$}

The fact that the Hamiltonian can be expressed as in Eq.2.2, in terms of the total spins $\mathbf{J}_{\alpha}^{2}$ of the plaquettes with crossings, leads naturally to the question of what component the ground state wavefunction has in sectors with each of the possible values for the quantum number $J$ for a given $\mathbf{J}_{\alpha}^{2}$. Expanding the ground state wavefunction $|\psi\rangle$ as

$$
|\psi\rangle=a_{0}|0\rangle+a_{1}|1\rangle+a_{2}|2\rangle,
$$

where $\mathbf{J}_{\alpha}^{2}|J\rangle=J(J+1)|J\rangle$ for $J=0,1$ or 2 , we determine the coefficients $a_{J}$ by measuring $\left\langle\psi\left|\mathbf{J}_{\alpha}^{2}\right| \psi\right\rangle$ and $\left\langle\psi\left|\mathbf{J}_{\alpha}^{4}\right| \psi\right\rangle$. Performing this calculation on the ground state for the 24 site cluster, we obtain $\left\langle\mathbf{J}_{\alpha}^{2}\right\rangle=0.83534$ and $\left\langle\mathbf{J}_{\alpha}^{4}\right\rangle=2.01968$ for every plaquette $\alpha$. This yields $\left|a_{0}\right|^{2}=0.611,\left|a_{1}\right|^{2}=0.374$, and $\left|a_{2}\right|^{2}=0.015$. For comparison, a covering of the lattice with nearest-neighbor singlets yields $\left|a_{0}\right|^{2}=0.25,\left|a_{1}\right|^{2}=0.75$ and $\left|a_{2}\right|^{2}=0.0$. Since our measured value of $\left|a_{2}\right|^{2}$ is small, we conclude that the ground state lies almost entirely within the subspace of states in which every plaquette with crossing contains a nearest-neighbor singlet. In addition, it is clear that resonance between different such singlet coverings must make a central contribution to the ground state energy. These results may serve as a constraint in future attempts to construct an approximate dimer description of the ground state, for instance along the lines of Ref. [34.

\section{Non-magnetic states in the spin gap}

The presence of a large number of non-magnetic excited states in the spin gap is a feature that the SLWC shares with the KHAF. These states are spread in energy across the spin gap, and the lowest of them defines the smallest excitation energy in the system: the singlet gap. The dependence of the singlet gap on cluster size 


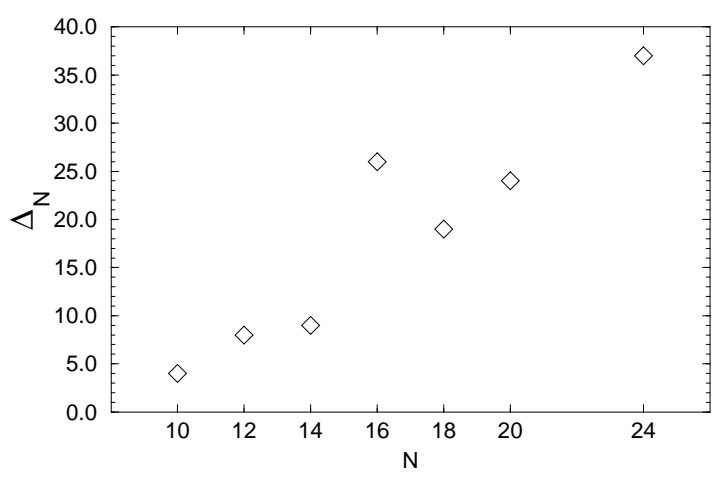

FIG. 8. The number of non magnetic $(S=0)$ states in the spin gap plotted versus system size.

is shown in Fig. 7. The gap is broadly decreasing with increasing cluster size, but it is unclear from these data whether it is non-zero or vanishing in the thermodynamic limit. In any case, it is very much larger than the singlet gap on kagomé clusters of similar size: we find for the SLWC with $N=24$ a singlet gap of 0.397 , to be contrasted with a singlet gap of $3.02 \times 10^{-3}$ for the $N=27$ kagomé cluster [7].

The total number of singlet states within the spin gap is large and grows with cluster size. In Figure 8 we show the number of states, $\Delta_{N}$, in the spin gap for each cluster size studied. The large, anomalous jump in $\Delta_{N}$ for the 16 site cluster is, presumably, a finite size effect particular to that sample. A comparison can be made with results for the KHAF described in Ref. [7]. At $N=24, \Delta_{N}=$ 37 for the SLWC and $\Delta_{N}=34$ for the KHAF, while more generally for the KHAF $\Delta_{N}$ grows with (even) $N$ as $1.14^{N}$.

\section{E. Ground state correlations}

Finally, we report on the measurements of the ground state correlation functions for the SLWC. We have calculated spin-spin and spin Peierls correlation functions for the 24 site cluster.

As expected both from the absence of quasi-degenerate joint states and from the large spin gap, the behavior of the spin-spin correlation function suggests that the SLWC does not have Néel order. Values are give in Table [1]. Correlations fall off rapidly with distance and are much smaller in magnitude than in comparison systems which are believed to have Néel order (for example, the spin-1/2 Heisenberg antiferromagnet on the triangular lattice, studied in a 36 site cluster in References [2] and [3]). Spin-spin correlations on the SLWC are similar in magnitude, but slightly larger than, the spin-spin correlations on the same sized cluster for the KHAF [5,6].

An alternative to Néel order as a pattern for symmetry breaking is spin Peierls or dimer order. To examine
TABLE II. Spin-spin correlations for the 24 site cluster. Correlations are measured between spins at various separations, $r$, from spin 9 (as labeled in Figures 9, 10, and 11). The nearest-neighbor distance is taken to be unity.

\begin{tabular}{ccc}
\hline \hline$n$ & $r$ & $\left\langle\mathbf{S}_{9} \cdot \mathbf{S}_{n}\right\rangle$ \\
\hline 15 & 1 & -0.378658 \\
14 & $\sqrt{2}$ & 0.048430 \\
16 & $\sqrt{2}$ & -0.081253 \\
21 & 2 & 0.131233 \\
20 & $\sqrt{5}$ & 0.048430 \\
19 & $2 \sqrt{2}$ & 0.002023 \\
23 & $2 \sqrt{2}$ & 0.002023 \\
12 & 3 & -0.002202 \\
\hline \hline
\end{tabular}

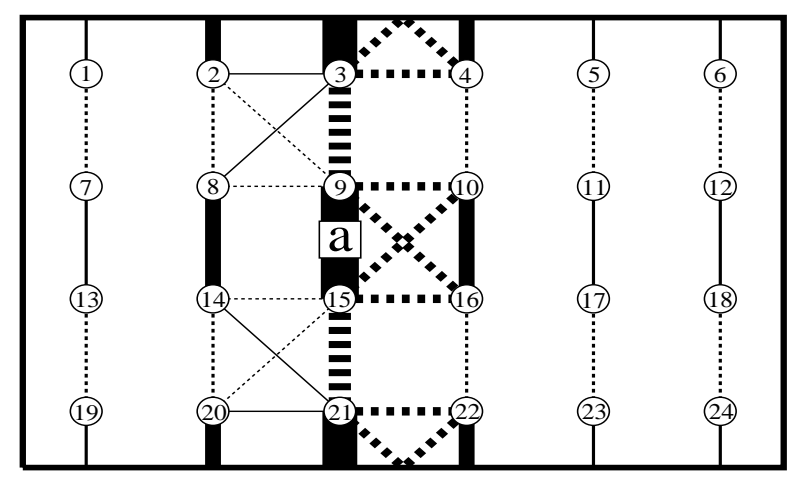

FIG. 9. A coarse-grained pictorial representation of the spin Peierls correlation functions measured for the 24 site cluster relative to the bond labeled $a$. Line widths are scaled with the magnitude of the correlation function. Positive correlations are given a solid line while negative correlations are represented with a dashed line. Correlations that are less than $8 \%$ of the on-bond correlation are omitted. The values of the correlation functions are given in Table III.

this possibility, we measure correlations between bond operators $D_{a}=\mathbf{S}_{i} \cdot \mathbf{S}_{j}$, where the bond $a$ joins nearestneighbor spins $i$ and $j$. We evaluate the connected correlation function

$$
C_{P}(a, b)=\left\langle\frac{1}{2}\left(D_{a} D_{b}+D_{b} D_{a}\right)\right\rangle-\left\langle D_{a}\right\rangle\left\langle D_{b}\right\rangle .
$$

The results are displayed in Figures 9, 10 and 11, and listed in Table III. The behavior of this correlation function is clearly influenced by finite cluster size and periodic boundary conditions. It is striking nevertheless that the correlation length measured along the long side of the cluster is no more than one lattice spacing. The magnitudes of spin Peierls correlations are comparable to those reported for the KHAF [5.6. 


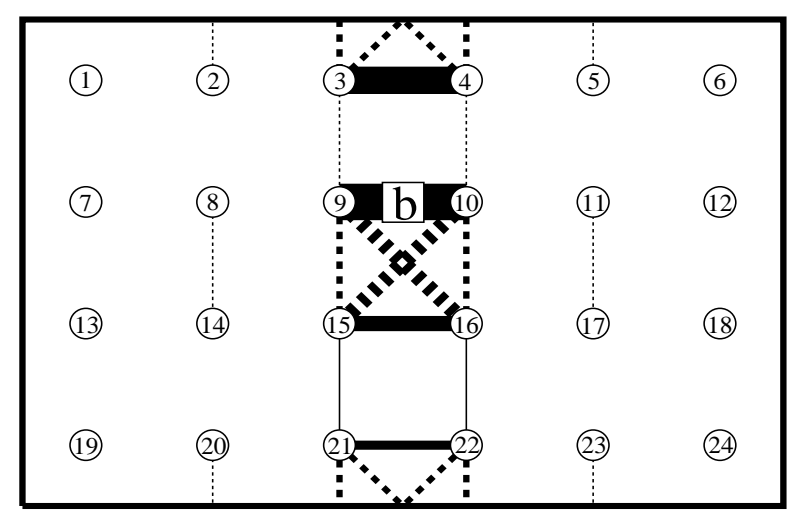

FIG. 10. Spin Peierls correlations measured relative to bond $b$ and represented as described in the caption of Figure 9 .

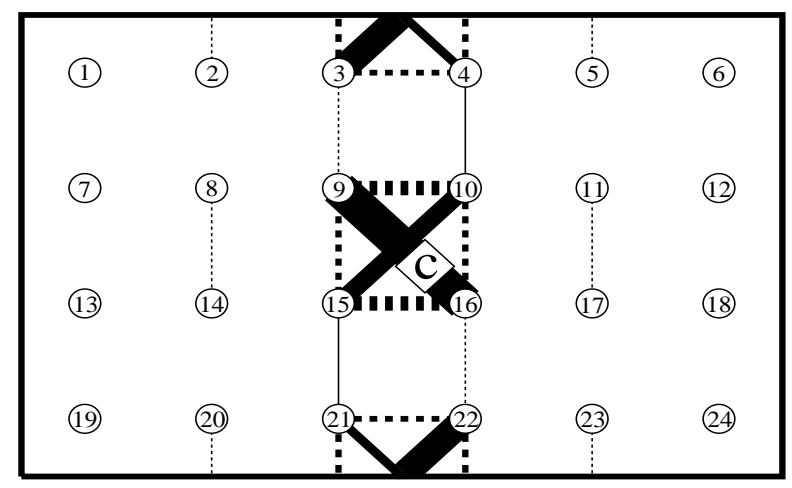

FIG. 11. Spin Peierls correlations measured relative to bond $c$ and represented as described in the caption of Figure 9 .
TABLE III. Spin Peierls correlations in the 24 site cluster of the SLWC. Spin Peierls correlations are measured between the bonds labeled $a, b$, and $c$, and all other bonds, $y$, in the cluster as pictured in Figures 9, 10, and 11. A bond $y$ is represented in the table by a pair numbers which label the two sites joined by $y$. Site numbers are as given in the aforementioned figures.

\begin{tabular}{cll}
\hline \hline$C_{P}(a, y)$ & bonds $y$ & \\
\hline 0.233447 & $(9,15)$ & \\
0.193017 & $(3,21)$ & \\
-0.110574 & $(3,9),(15,21)$ & \\
0.083771 & $(10,16)$ & \\
0.082317 & $(4,22)$ & \\
0.079108 & $(8,14),(2,20)$ & \\
-0.051080 & $(9,16),(9,10),(10,15),(15,16)$ & \\
-0.049528 & $(3,4),(3,22),(4,21),(21,22)$ & \\
0.034932 & $(1,19),(5,23),(7,13),(11,17)$ & \\
0.031582 & $(6,24),(12,18)$ & \\
-0.026960 & $(2,8),(4,10),(14,20),(16,22)$ & \\
-0.026735 & $(5,11),(17,23)$ & \\
-0.020674 & $(1,7),(13,19)$ & $(3,9),(16,22)$ \\
-0.020024 & $(6,12),(18,24)$ & $(2,20),(5,23)$, \\
0.020004 & $(2,3),(3,8),(14,21),(20,21)$ & \\
-0.018660 & $(2,9),(8,9),(14,15),(15,20)$ & \\
\hline$C_{P}(b$ or $c, y)$ & $y$ for $C_{P}(b, y)$ & $(11,17)$ \\
\hline 0.221524 & $(9,10)$ & $(3,16)$ \\
0.155176 & $(3,4)$ & $(9,10,15),(15),(21,16)$ \\
0.109339 & $(15,16)$ & $(10,16)$ \\
-0.101267 & $(9,16),(10,15)$ & \\
0.076887 & $(21,22)$ & \\
-0.056178 & $(3,22),(4,21)$ & \\
-0.051080 & $(9,15),(10,16)$ & \\
-0.049528 & $(3,21),(4,22)$ & \\
0.020004 & $(15,21),(16,22)$ & \\
-0.018660 & $(3,9),(4,10)$ & \\
-0.017738 & $(2,20),(5,23)$, & \\
& $(8,14),(11,17)$ & \\
\hline \hline
\end{tabular}




\section{DISCUSSION}

In summary, the results described in Sec. III combine to demonstrate that the SLWC has a ground state with unbroken spin rotation symmetry. The lack of Néel order is signaled by the absence of quasi-degenerate joint states, by the magnitude and cluster size dependence of the spin gap, and most directly by the spin-spin correlation function itself. It is less clear whether or not the translational symmetry of the model is broken in the ground state, although the short range of the spin Peierls correlations along one direction in Figures 9 11 is suggestive of unbroken translational symmetry. If translational symmetry is broken, one might expect either columnar dimerization or plaquette resonating valence bond states, as considered for the $J_{1}-J_{2}$ model near $J_{1} / J_{2}=2$ 14 $\left.16,19,20\right]$. Since only a few examples are known of two-dimensional Heisenberg models with magnetically disordered ground states, it is a problem of some theoretical interest to decide between these alternatives and, especially, to understand the nature of the low-lying singlet excitations.

\section{ACKNOWLEDGMENTS}

We are grateful to P. Lecheminant and G. Misguich for very helpful discussions. S.E.P. would like to thank the Rhodes Trust and the Alfred P. Sloan Foundation for financial support. The work was also supported in part by EPSRC grants GR/J78327 and GR/M56234. Some preliminary results for small (up to 12-site) clusters were obtained using TITPACK v. 2 (C) Hidetoshi Nishimori.

[1] A. P. Ramirez, Annu. Rev. Mater. Sci. 24, 453 (1994); P. Schiffer and A. P. Ramirez, Comments Cond. Mat. Phys. 18, 21 (1996); M. J. Harris and M. P. Zinkin, Mod. Phys. Lett. B 10, 417 (1996); C. Lhuillier, P. Sindzingre, and J.-B. Fouet, cond-mat/0009336 (2000).

[2] B. Bernu, P. Lecheminant, C. Lhuillier, and L. Pierre, Phys. Rev. B 50, 10048 (1994).

[3] L. Capriotti, A. E. Trumper, and S. Sorella, Phys. Rev. Lett. 82, 3899 (1999); A. E. Trumper, L. Capriotti, and S. Sorella, Phys. Rev. B 61, 11529 (2000).

[4] C. Zeng and V. Elser, Phys. Rev. B 42, 8436 (1990).

[5] J. T. Chalker and J. F. G. Eastmond, Phys. Rev. B 46, 14201 (1992).

[6] P. W. Leung and V. Elser, Phys. Rev. B 47, 5459 (1993).

[7] P. Lecheminant, B. Bernu, C. Lhuillier, L. Pierre, and P. Sindzingre, Phys. Rev. B 56, 2521 (1997).

[8] C. Waldtmann, H.-U. Everts, B. Bernu, P. Sindzingre, C. Lhuillier, P. Lecheminant, and L. Pierre, Euro. Phys. J. B 2, 501 (1998).
[9] B. Canals and C. Lacroix, Phys. Rev. Lett. 80, 2933 (1998).

[10] R. Moessner and J. T. Chalker, Phys. Rev. B 58, 12049 (1998).

[11] E. H. Lieb and P. Schupp, Phys. Rev. Lett. 83, 5362 (1999).

[12] P. Chandra and B. Douçot, Phys. Rev. B 38, 9335 (1988).

[13] F. Figueirido, A. Karlhede, S. Kivelson, S. Sondhi, M. Rocek, and D. S. Rokhsar, Phys. Rev. B 41, 4619 (1989).

[14] E. Dagotto and A. Moreo, Phys. Rev. Lett. 63, 2148 (1989).

[15] M. P. Gelfand, R. R. P. Singh, and D. A. Huse, Phys. Rev. B 40, 10801 (1989).

[16] R. R. P. Singh and R. Narayanan, Phys. Rev. Lett. 65, 1072 (1990).

[17] H. J. Schultz and T. A. L. Ziman, Europhys. Lett. 18, 355 (1992).

[18] H. J. Schultz, T. A. L. Ziman, and D. Poilblanc, J. Phys. I France 6, 675 (1996).

[19] L. Capriotti and S. Sorella, Phys. Rev. Lett. 84, 3173 (2000).

[20] V. N. Kotov, J. Oitmaa, O. Sushkov, and Z. Weihong, Phys. Rev. B 60, 14613 (2000).

[21] M. S. L. du Croo de Jongh, J. M. J. van Leeuwen, and W. van Saarloos, Phys. Rev. B 62, 14844 (2000).

[22] O. P. Sushkov, J. Oitmaa, and Z. Weihong, Phys. Rev. B 63, 104420 (2001).

[23] B. Canals, cond-mat/0102233 (2001).

[24] G. Misguich, B. Bernu, C. Lhuillier, and C. Waldtmann, Phys. Rev. Lett. 81, 1098 (1998).

[25] G. Misguich, C. Lhuillier, B. Bernu, and C. Waldtmann, Phys. Rev. B 60, 1064 (1999).

[26] W. LiMing, G. Misguich, P. Sindzingre, and C. Lhuillier, Phys. Rev. B 62, 6372 (2000).

[27] P. W. Leung and K. J. Runge, Phys. Rev. B 47, 5861 (1993).

[28] P. W. Anderson, Phys. Rev. 86, 694 (1952).

[29] D. S. Fisher, Phys. Rev. B 39, 11783 (1989).

[30] M. Gross, E. Sánchez-Valasco, and E. D. Siggia, Phys. Rev. B 40, 11328 (1989).

[31] M. Gross and E. Sánchez-Velasco, Phys. Rev. B 39, 2484 (1989).

[32] H. Neuberger and T. Ziman, Phys. Rev. B 39, 2608 (1989).

[33] S. Tang and J. E. Hirsch, Phys. Rev. B 39, 4548 (1989).

[34] M. Mambrini and F. Mila, Euro. Phys. J. B 17, 651 (2000). 